\title{
ÉDITORIAL
}

\section{Y a-t-il un médecin dans la pharmacie ?}

\author{
P. Goetz \\ (C) Lavoisier SAS 2016
}

On croyait la phytothérapie morte et enterrée. Et bien non ! Plus, à titre personnel, j'ai envie d'aller vers la retraite, plus je reçois des patients qui me réclament ce mode de traitement. L'engouement populaire et médical des années de mouvance écologique de 80 à 90 , lors de la première révolution verte, a été remplacé par une demande accrue des patients, lassés par les médicaments conventionnels, par la mauvaise presse des génériques et le discrédit que l'industrie pharmaceutique a réussi à attirer sur elle.

Actuellement l'informatique offre une nouvelle donne, mais très mauvaise dans les faits : la facilité d'obtenir des huiles essentielles sur les étagères de n'importe quelle boutique de produits naturels et pire encore sur internet. La phyto(aroma)thérapie est passée du contrôle pharmaceutique à des listes interminables de plantes-compléments alimentaires acceptées, mais peut-être pas acceptables.

Nous saluons les médecins et pharmaciens qui fréquentent les $\mathrm{DU}^{1}$ et qui se proposent de passer des $\mathrm{DPC}^{2}$ où figurent des questionnaires concernant la phyto-aromathérapie.

Quand nous commençâmes sous la tutelle du docteur Paul Belaiche à assurer un enseignement de phytothérapie à Paris XIII, nous étions 7 enseignants médecins sur 10. Aujourd'hui, le rapport doit être inverse.

Politiques et études médicales ont essayé de faire table rase des $\mathrm{MEP}^{3}$ en général et de la phytothérapie en particulier. On y revient car si la médecine de pointe est indispensable, beaucoup de malades restent sur la touche avec leurs problèmes et n'ont aucun secours. Il y a de nombreuses maladies orphelines, mais beaucoup plus de "patients orphelins ", qui ont besoin d'une aide.

Rappelons un concept de base : le médecin fait un diagnostic, propose un traitement et suit le cours de la maladie ; le pharmacien assure au patient l'accès au bon médicament à la bonne dose. L'accord doit être harmonieux entre ces deux partenaires indispensables. Nous saluons tous les pharmaciens qui prennent le temps de se former en phytothérapie, car avant de consulter son médecin, le malade va souvent demander au pharmacien un remède qui sort du cadre de la prescription médicale habituelle.

Rappelons que si un médicament-molécule a une série limitée d'indications, les plantes et les huiles essentielles contiennent de nombreux constituants chimiques, de potentiels principes actifs, pharmaciens ou médecins se souviennent que l'harpagophytum est anti-inflammatoire, mais également une drogue capable de stimuler la fonction hépatobiliaire, et peut associer ces deux activités dans une affection comme une hépatite par surcharge biliaire, pathologie pour laquelle la médecine conventionnelle est muette.

Nos phytomédicaments ne sont pas non plus tout puissants mais en général au moins ils soulagent! Il existe par ailleurs, mais pas en France, des études qui cherchent à évaluer l'interaction médicamenteuse entre médicament de synthèse et phytomédicaments. Les plus souvent cités sont bien sûr le millepertuis et le pamplemousse. La prise de pamplemousse semble être d'ailleurs plus nuisible que celle du millepertuis. C'est ici que se joue le rôle de conjugaison entre le pharmacien informé et le phytothérapeute : ne cherchons pas toujours l'effet nocif, reconnaissons aussi les interactions positives comme, par exemple, de pouvoir diminuer les antidiabétiques oraux avec l'instauration de phytomédicaments qui agissent en inhibant l' $\alpha$-glucosinase. Les deux effets s'ajoutent sans perturbations pour le patient.

La drogue végétale n'est pas une substance inerte, sachons revue après revue, apprendre le b.a.-ba, du plus simple aux associations les plus complexes.

Pharmaciens comme médecins, souvenons-nous que nous n'appliquons pas une science, mais pratiquons un art.

P. Goetz $(\bowtie)$

Enseignant au DU de Phytothérapie (Faculté de médecine,

Paris XIII), F-93017 Bobigny cedex, France

e-mail : paul.goetz@wanadoo.fr

\footnotetext{
${ }^{1}$ Diplômes universitaires

${ }^{2}$ Développement professionnel continu

${ }^{3}$ Modes d'exercice particulier
} 